\title{
Cournot Game of ISP under Net Neutrality
}

\author{
Mohamed El Amrani ${ }^{1}$, Hamid Garmani ${ }^{2}$, Mohamed Baslam ${ }^{3}$, \\ Brahim Minaoui ${ }^{4}$ and Rachid El Ayachi ${ }^{5}$ \\ 1,2,3,4,5 Information Processing and Decision Support Laboratory, Faculty of \\ Sciences and Technics, Sultan Moulay Slimane University, Beni Mellal, Morocco \\ 1,2,3 \{med.el.amran, garmani.hamid, baslam.med\}@gmail.com, \\ ${ }^{4}$ bra_min@yahoo.fr, ${ }^{5}$ rachid.elayachi@usms.ma
}

\begin{abstract}
Network neutrality has been a very sensitive topic of discussion all over the world. In this paper, we study the competition between ISPs under two-regime non-neutral network and neutral network. We interpret non-neutral network when an internet service provider is privileged by the Content Provider (CP) in order to propose a high quality of service for a given content. Competition among ISPs modeled as a Cournot game. Noncooperative game is utilized to study the interactions among ISPs. On the one hand, each ISP decide his policies of Quality of Service $(Q o S)$ in order to maximize his profit. In turn, the end-users demand for the service of an ISP depends not only on their strategies, but also upon those proposed by all of its competitors. A unique Nash equilibrium is established, through rigorous mathematical analysis. Furthermore, we introduce a learning algorithm that may lead ISPs to learn their strategic QoS in a complete distributed manner. In order to quantify how efficient is the Nash equilibrium point, a detailed analysis of the Price of Anarchy is adopted to ensure the performance of the system at equilibrium. Extensive simulations show the convergence to the Nash equilibrium and give some insights on how the game parameters may vary the ISPs revenue, demand and policies.
\end{abstract}

Keywords: Net Neutrality, Economic Model of Networks, Game Theory, Nash Equilibrium, Quality of Service(QoS), Social Welfare

\section{Introduction}

The Internet is a ubiquitously available platform for information, entertainment and communication. Over the years, the position of ISPs (Internet Service Providers) has become one of an essential gatekeeper, which puts them in control of the information flow on the web. Network neutrality has become a very hot topic in the past few years [12][13][14][16][1], at the same time from political, economic, and daily-life points of view, because it may refashion the Internet business model and in general the telecommunications vision and future. In short, the dispute started in the 2000s between ISPs and major content providers (CPs). Net neutrality is defined as the concept in which ISPs are obliged to treat all data streams equally, independent of which application, service, device, sender or receiver is involved.

Game theory [7] is the mathematical study of the behavior of decision-makers (players) whose decisions affect each other. This theory models the interactions between players with conflicting objectives. It has been applied its application in many fields where the strategies for each player depend on the actions of all. Nash Equilibrium [17] is one of the fundamental concepts of game theory which describes the equilibrium when each players strategy is a best response to all other strategies.

Received (April 3, 2018), Review Result (June 4, 2018), Accepted (June 7, 2018) 
It is a solution where the optimal outcome of a game is that in which given what all players are doing, no one player can benefit by changing.

There exists a recent flourishing literature dealing with network neutrality modeling and analysis, see among others [5][18] [19][22][15]. But those papers mainly discuss how revenue should be shared among providers (ISPs and CPs), side payments or off-network pricing through analytical models. The authors in [4] study a competition between ISPs as Bertrand competition under two cases: case of neutral network and case of non-neutral network. In a Bertrand competition, ISPs compete on price -with each one choosing its price independently and at the same time- and supply QoS demanded at those prices.

In this paper we consider a Cournot competition between ISPs. In economics [21], Cournot competition/game is a model of competition in which firms (internet service providers in our case) compete on quantity (i.e., quality of service) they produce-which they decide independently and at the same time-based on their cost of production, represented the cost of bandwidth. In a Cournot game, the market price is set at a level such that demand equals the total quantity produced by all firms. In other words, the service price is determined by the supply and demand for services in the market.

In our model, we use a Cournot game model to characterize the competition between ISPs, as we assume they produce substitutable QoS and the price of these QoS is essentially determined by competition, market supply and demand. In other words, due to competition, different ISPs have limited ability to choose their own prices; and at equilibrium, they converge to the same price and consequently determine the amount of QoS.

The main contributions of this work can therefore be summarized as follows:

- We include quality of service in competition between ISPs for end-users in the demand markets.

- We model competition between ISPs based on one market parameter quality of service, as well each ISP want to maximize its utility. We formulate this competitive problem between ISPs as non-cooperative game.

- We analytically prove the existence and uniqueness of Nash equilibrium in noncooperative game between ISPs in neutral and non-neutral network, which means that there exists a stable state where all ISPs do not have an incentive to change their strategies.

- Numeric analysis shows the effect of neutral and non-neutral regime on ISPs policies.

The rest of the paper is organized as follows. In Section 2, we describe the model of discord between internet service providers and their subscribers, also, we present definition of Nash equilibrium. In Sections 3 and 4 we provide theorems for existence and uniqueness of equilibria respectively in case of net neutrality and non-neutrality. Section 5 presents numerical study to validate our claims, Section 6 presents a study of the impact of competition and Section 7 concludes the paper.

\section{Problem Modeling}

Our economic model is composed of a content provider (CP) and $N$ Internet Service Providers (ISP) in competition on a set of customers. The figure 1 illustrates a typical example of systems with a single content provider $(C P)$ and several Internet Service providers which each serves a set of end users $(E U)$.

Here, the $C P$ provide multimedia contents (e.g., music streaming, VOIP, ...). This multimedia content is transported and placed at the disposal of the EUs on the physical infrastructure of $I S P_{i}$. Under the neutral network configuration, EUs and the content provider pay only for their direct access. On one hand, and according to 
the rate of the own demand for $I S P_{i}, C P$ decided to invest and allocate bandwidth $\Phi_{c}^{i}$ to it. This can be considered as a preferred contract is between $I S P_{i}$ and $C P$. Yet, as the demand of $I S P_{i}$ increases as it becomes more beneficial to $C P$ to invest more and more. On the other, each $I S P_{i}$ charges to its customers an amount $p_{i}$ per unit of traffic. Moreover, each $I S P_{i}$ allocates a bandwidth $\Phi_{i}$ to its customers to guarantee their promised $\operatorname{QoS} \tilde{q}_{i}$.

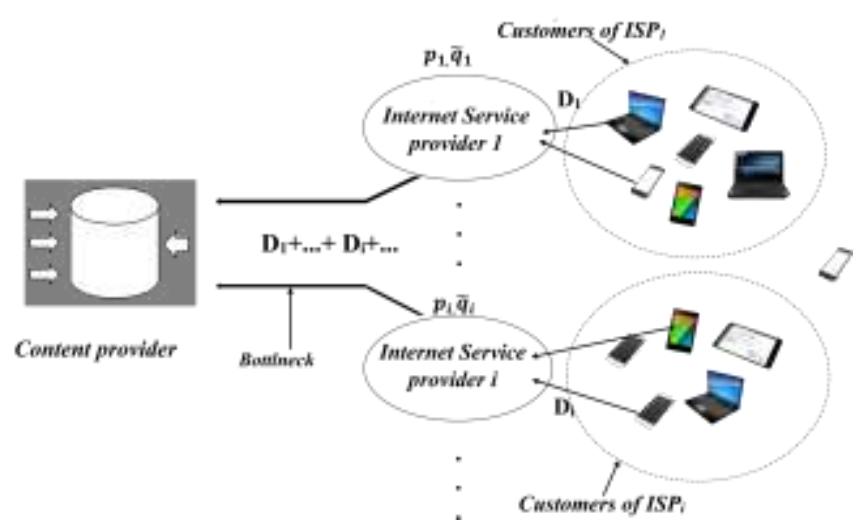

Figure 1. Single Content Provider and Several Internet Service Providers

In the rest, we consider the following notations: $p=\left(P_{1}, \ldots, P_{j}, ..\right)$ and $\tilde{q}=\left(\tilde{Q}_{1}, \ldots, \tilde{Q}_{j}, \ldots\right)$ for the charged price vector and the promised $Q o S$ respectively.

\subsection{Demand Model}

We consider that the demand function $D_{i}($.$) for the I S P$ services is linear with respect to price ( $p_{i}$ ) fixed by the $I S P_{i}$ and promised $e 2 e Q o S$, see [2] and [4] [9]. This demand also depends on price $p_{-i}$ and $e 2 e Q o S$ of competitors, namely, the demand function depends on $p$ and $\tilde{q}$. Naturally, the demand function $D_{i}$ is decreasing w.r.t $p_{i}$ and increased w.r.t $p_{-i}$, while it is increasing w.r.t $\tilde{q}_{i}$ and it is decreasing, w.r.t $\tilde{q}_{-i}$. We consider that the demand functions w.r.t the $I S P_{i}$ service can be written as follows:

$$
D_{i}(p, \tilde{q})=a_{i}-\alpha_{i}^{i} p_{i}+\beta_{i}^{i} \widetilde{q}_{i}+\sum_{j, j \neq i}\left\lfloor\alpha_{i}^{j} p_{j}-\beta_{i}^{j} \tilde{q}_{j}\right\rfloor,
$$

where $a_{i}$ is a positive constant used to ensure the non-negativity of the demands on the feasible region.

Considering that $\alpha_{i}^{j}$ and $\beta_{i}^{j}$ are positive constants and standardized, which represent respectively the the $I S P_{i}$ sensitivity to the price and the $Q o S$ of the $I S P_{j}$, as follows:

$$
\sum_{j=1}^{N} \alpha_{i}^{j}=1 \text { and } \sum_{j=1}^{N} \beta_{i}^{j}=1, \quad i=1, \ldots, N .
$$

Assumption 1 For any e2e QoS profile, the e2e QoS mutual sensitivities satisfy:

$$
\beta_{i}^{i} \geq \sum_{j, j \neq i} \beta_{i}^{j}, \forall i, j=1, \ldots, N .
$$


The assumption 1 is realistic and has no impact on the field of applicability of this work. Indeed, considering that the influence of $e 2 e Q o S$ promised by the $I S P_{i}$ on its gains more weight relative to the sum of the influences of promised $e 2 e Q o S$ by ISPs competitors on $I S P_{i}$ gains. This condition could then take into account the presence of loyalties of customers and/or partial knowledge of $e 2 e Q o S$ of competitors. Moreover, this assumption is a reasonable condition which guarantees supermodularity of the game and So the uniqueness of Nash equilibrium.

Assumption 2 For any vector quality of service of competitors, the total demand $D(p, \tilde{q})=\sum_{i=1}^{N} D_{i}(p, \tilde{q})$ is an increasing function w.r.t the individual e2e QoS $\tilde{q}_{i}$ of the $I S P_{i}$.

Considering the desire of paying customers, it becomes plausible to consider that the total demand is increasing depending on individual $e 2 e Q o S$ fixed by $I S P_{i}$. When the $I S P_{i}$ decides to decrease its quality of service, attached customers would be migrated and subscribe with its competitors or decide to unsubscribe. In other words, this assumption says that the effect of the $e 2 e Q o S$ individual is still higher than the overall perceived influence from competitors. An important characteristic is that the variation of the total demand, compared to individual $e 2 e Q o S$ is very low, because few customers decide to unsubscribe completely.

Remark 1 From the assumption 2, we deduce that the first derivative of total demand w.r.t $\tilde{q}_{i}$ is positive, then, the e2e QoS mutual sensitivities satisfy:

$$
\beta_{i}^{i}-\sum_{j, j \neq i} \beta_{i}^{j} \geq 0, \forall i, j=1, \ldots, N .
$$

Assumption 3 We assume that the influence of QoS e2e for an ISP $P_{i}$ on its demand is inversely proportional to the number of its competitors $(N)$.

$$
\beta_{i}^{i}-\sum_{j, j \neq i} \beta_{i}^{j} \leq \frac{1}{N}, \forall i, j=1, \ldots, N .
$$

The number of competitors can influence the demand for a particular $I S P_{i}$ in a remarkable manner when the number becomes bigger and vice versa, i.e. of the sum of the mutual sensitivities to its competitors quality of service become very close to that of the $I S P_{i}$, so that deference sensitivities can be bounded by the inverse of the total number of these competitors.

Remark 2 From the assumption 3 and remark 1, we can say:

$$
0 \leq \beta_{i}^{i}-\sum_{j, j \neq i} \beta_{i}^{j} \leq \frac{1}{N}, \forall i, j=1, \ldots, N .
$$

\subsection{Utility Model}

We turn now to take the utility function of each ISPs and the single $C P$. Let $q_{i}$ (endto-end quality of service) the real quality perceived by the $E U$ s of $I S P_{i}$, (In this case $\tilde{q}_{i}$ 
is the $Q o S$ promised / advertised by the $I S P_{i}$ for end users). The net revenue of $I S P_{i}$ is exactly the difference between its total revenue and its expenses. These latter correspond to the sum of the costs to cover the costs of bandwidth $\Phi_{i}$ and a certain penalty if it does not meet promised quality $\tilde{q}_{i}$. Thus, net revenue is given by as following:

$$
U_{i}^{I S P}(p, \tilde{q})=p_{i} D_{i}(p, \tilde{q})-\vartheta_{i} \phi_{i}+\theta_{i}\left(q_{i}-\tilde{q}_{i}\right),
$$

where $\vartheta_{i}$ and $\theta_{i}$ are positive constants.

\subsection{Game Analysis}

In the rest of this work, we will analyze the game that arises in both the establishment of neutrality and non-neutrality. The players are the ISPs that must to define their strategies for the price $\left(p_{i}\right)$ and the promised quality of service $\left(\tilde{q}_{i}\right)$. The concept of neutrality is considered assuming that the $C P$ distributes its bandwidth as the processor sharing principle. In another hand, the non-neutrality scenario is taken into account by assuming that there is one (more) specific $I S P(\mathrm{~s})$ (namely $I S P_{1}$ ) who signed a contract with the $C P$, in order to reserve him a amount of bandwidth to ensure the promised quality of service $\left(\tilde{q}_{1}\right)$. In this context, the competitors of $I S P_{1}$ equally share the remaining bandwidth as if we have neutrality case by taking into account the topology when we do not have the $I S P_{1}$.

Definition of Nash equilibrium of the $Q o S$ game: We consider a game of strategic form of N-players

$$
\Gamma=\left\{\mathrm{N}, \tilde{Q}_{1}, \ldots, \widetilde{Q}_{N}, U_{1}^{I S P}, \ldots, U_{N}^{I S P}\right\},
$$

where $\tilde{Q}_{i}$ is the set of $Q o S$ strategies of player $i$ and $U_{i}^{I S P}$ its utility function.

Definition 1 Nash equilibrium specifies a strategy $\tilde{q}_{i}^{*} \in \tilde{Q}_{i}$ for each player $i$ (with $i=1, \ldots, N$ ) in such a way that:

$$
U_{i}^{I S P}\left(p, \tilde{q}^{*}\right)=\max _{\tilde{q}_{i} \in \tilde{Q}_{i}} U\left(p, \tilde{q}_{1}^{*}, \ldots, \tilde{q}_{i-1}^{*}, \tilde{q}_{i}, \tilde{q}_{i+1}^{*}, \ldots, \tilde{q}_{N}^{*}\right),
$$

When the vector of price parameters, $p$, of all providers is fixed to a certain predetermined point.

Below, we analyze the competitive qualities of service for $N I S P$ s that maximize their utilities. To do so, we demonstrate the existence and uniqueness of the game equilibrium between $N I S P \mathrm{~s}$, after we calculate the equilibrium point. To analyze equilibrium of the game, we need to find properties on the utility function which require that we describe the expression in both cases.

\subsection{Learning Nash Equilibrium}

In [2], [18], [3], [6], the main concern was the search for the equilibrium situations (namely Nash). Lately researchers are questioning the need for convergence of a learning algorithm to a Nash equilibrium, there are several reasons for this. First, there may the multiple equilibria in a game, and it can not any method for coordinating choice of agent.

Learning algorithms resemble the behavior of competitors in many naturally arising games, and thus results, on the convergence or non-convergence properties of such dynamics may inform our understanding of the applicability of Nash equilibria as a 
plausible solution concept in some settings. In the reality, when every ISP tries to maximize its revenue, it is the most natural to accept Nash equilibrium as the optimal solution concept. In Nash equilibrium, each ISP's strategy is the best response to the other ISP s's strategies. Thus no ISP can gain from unilateral deviation.

Algorithm 1 Best Response algorithm

1. Initialization of $Q o S$ vectors;

2. For each $I S P_{i} i \in \mathrm{N}$ at iteration t:

$$
\tilde{q}_{i}^{t+1}=\underset{\tilde{q}_{i} \in \tilde{Q}_{i}}{\operatorname{argmax}}\left(U_{i}\left(p, \tilde{q}^{t}\right)\right)
$$

\section{Neutral Networks}

Remember that for the sharing capability of ISPs under the Process Sharing ( $P S$ ) principles, $\Phi_{i}$ is given by combining both the expression of Delay in the links between $I S P_{i}$ and $E U \mathrm{~s} \mathrm{(L.} \mathrm{Kleinrock} \mathrm{[10])}$

$$
\operatorname{Delay}_{i}^{u}=\frac{1}{\Phi_{i}-D_{i}(p, \tilde{q})},
$$

and the expression of the quality of service promised by the $I S P_{i}$ to end users ( $E U \mathrm{~s}$ )

$$
\tilde{q}_{i}=\frac{1}{\text { Delay }_{i}^{u}+c_{i}}
$$

thus:

$$
\Phi_{i}=D_{i}(p, \tilde{q})+\frac{\tilde{q}_{i}}{1-\tilde{q}_{i} c_{i}},
$$

where $c_{i}$ is the expected delay in the link between $I S P_{i}$ and $C P$.

The end-to-end delay (denoted Delay ${ }_{c}^{u}$ ) experienced by end users of the $I S P_{i}$, namely, the cumulative delay on both links $E U-I S P_{i}$ and $I S P_{i}-C P$, depends on: the total demand for services of $C P, D(p, \tilde{q})$, the demand for services transported by $I S P_{i}$, $D_{i}(p, \tilde{q})$, the bandwidth allocated by the $C P$ to $I S P_{i}, \Phi_{c}$, and $\Phi_{i}$ the bandwidth allocated by $I S P_{i}$ to its customers:

$$
\text { Delay }_{c}^{u}=\frac{1}{\Phi_{i}-D_{i}(p, \tilde{q})}+\frac{1}{\Phi_{c}-D(p, \tilde{q})},
$$

The real $Q o S$ perceived by end users of the $I S P_{i}$ is the inverse of the end-to-end delay,

$$
q_{i}=\frac{1}{\operatorname{Delay}_{c}^{u}}=\frac{\tilde{q}_{i}\left(\Phi_{c}-D(p, \tilde{q})\right)}{\tilde{q}_{i}+\left(1-\tilde{q}_{i} c_{i}\right)\left(\Phi_{c}-D(p, \tilde{q})\right)},
$$

By replacing $\Phi_{i}$ and $q_{i}$ by their expressions in equation ((6)), the utility function of $I S P_{i}$ can be written as:

$$
U_{i}^{I S P}\left(p, \tilde{q}_{i}\right)=\left(p_{i}-\vartheta_{i}\right) D_{i}(p, \tilde{q})-\frac{\vartheta_{i} \tilde{q}_{i}}{1-\tilde{q}_{i} c_{i}}+\theta_{i} \tilde{q}_{i}\left(\frac{\Phi_{c}-D(p, \tilde{q})}{\tilde{q}_{i}+\left(1-\tilde{q}_{i} c_{i}\right)\left(\Phi_{c}-D(p, \tilde{q})\right)}-1\right) .
$$


The question is, under general assumptions, when can we guarantee the existence and uniqueness of the equilibrium due through the ISPs? We consider that the quality of service is the only parameter of the game $((7))$ which arose when the price of all ISP $\mathrm{s}$ is fixed. Thus, under the assumption 1 and according to the remark 1, we have the following general result on uniqueness of quality of service based on Nash equilibrium for all $\mathrm{N}$ ISP s.

Lemma 1 (Existence) Considering the game of levels of quality of service which arose when the price vector is fixed for all ISPs, there exists in less one quality of service based Nash equilibrium $\tilde{q}^{*}$, of ISPs game.

Lemma 2 (Uniqueness) On the assumption 1 and according to the remark 1, the equilibrium point $\tilde{q}^{*}$, of the game of ISPs, which arises when the price vector is fixed to all ISP s, is unique.

under lemmas $(1,2)$, we deduce the following theorem:

Theorem 1 (existence and uniqueness) Consider the game of levels of quality of service(7) which arose when the price vector is fixed to all ISPs, under the assumptions 1, 3 and according to the remark 2, there is only one level of quality of service based Nash equilibrium $\tilde{q}^{*}$ of the ISPs game. (the proof is given by appendices 7.1 and 7.2)

\section{Non-Neutral Networks}

As we mentioned above (section 2), in non-neutral networks we assume that there is a specific ISP (i.e., ISP $P_{1}$ ) who signed a special contract with $C P$, to reserve for him a quantity of bandwidth to guarantee the promised quality of service $\tilde{q}_{1}$. Under a nonneutral system, the $I S P_{1}$ will guarantee the promised $Q o S$, and therefore, the expression of the value of $I S P_{1}$ becomes simpler since $\tilde{q}_{i}=q_{i}$ i.e., no penalty does not appear in the function utility of the $I S P_{1}$ :

$$
U_{1}^{I S P}(p, \tilde{q})=\left(p_{1}-\vartheta_{1}\right) D_{1}(p, \tilde{q})-\frac{\vartheta_{1} \tilde{q}_{1}\left(\Phi_{c}-D_{1}(p, \tilde{q})\right)}{\Phi_{c}-D_{1}(p, \tilde{q})-\tilde{q}_{1}} .
$$

In this context, the competitors of the $I S P_{1}$, equally share the remaining bandwidth. In the absence of the $I S P_{1}$, the network behaves as a neutral network where other ISPs are competing over the common bandwidth. The utility function of other internet service providers ( $I S P_{1}$ competitors) is given by equation (11).

Theorem 2 (existence and uniqueness) Consider the game of quality levels for services (7) that arises without net neutrality. Under the assumption 1 and according to the remark 1, there is one level of Nash equilibrium quality of service therefore the utility function of ISPs satisfies the properties of concavity and uniqueness. (the proof is given by appendix 7.3)

\section{Numerical Results}

We turn now to discuss how to take advantage of our analytical results. We propose to study numerically the market share game taking account of previous expressions of demand functions and utility of the ISPs. To illustrate, we consider two homogeneous 
ISPs looking to maximize their respective payoffs. In particular, we discuss the influence of the penalty factor $\theta_{i}$ and the bandwidth of the $C P$ in cases of neutrality and nonneutrality. The Figures 2 and 3 represent respectively the curves of the convergence to Nash equilibrium $e 2 e Q o S$ in both neutral and non-neutral network, it is clear that the best response dynamic algorithm 2.4 convergence to the unique Nash equilibrium $e 2 e$ $Q o S$, in both cases, we also notice the convergence speed is relatively fast (6 iterations for the neutral case and 5 iterations for the non-neutral case). So this simulation of the algorithm 2.4 is able to efficiently converge the Nash equilibrium $e 2 e Q o S$ in neutral and non-neutral network.

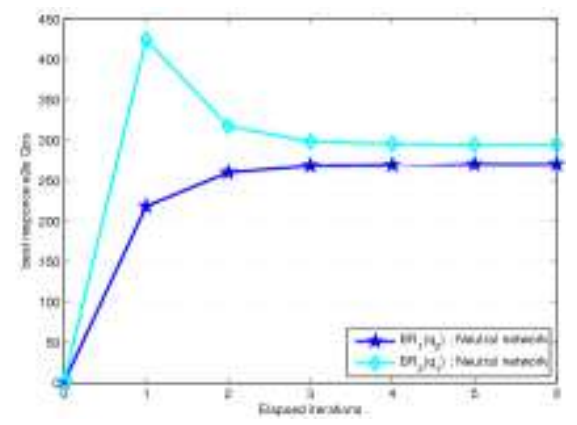

Figure 2. Neutral Network: Convergence to the Nash Equilibrium $e 2 e Q o S$

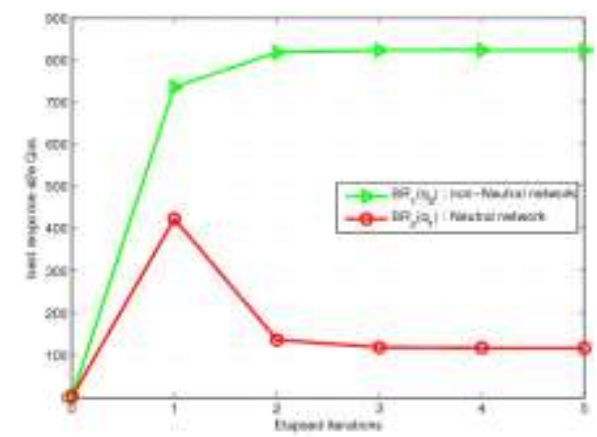

Figure 3. Neutral non-network: Convergence to the Nash Equilibrium e $2 e Q o S$

\subsection{Impact of the QoS Penality Factor}

The Figures 4 and 5 represent the variation of the $e 2 e Q o S$ at equilibrium and partitioning of the total demand at equilibrium points w.r.t penalty factor $\theta$, we note for null values penalty factor, all ISPs, over neutrality or non-neutrality cases, have the same results of $e 2 e Q o S$ (resp. of the demand partitioning). And as we see, the changes in the $e 2 e Q o S$ is similar to those of demand partitioning, which is normal since the demand $D_{i}$ (.) of $I S P_{i}$ is proportional to its $e 2 e \operatorname{QoS}\left(\tilde{q}_{i}\right)$. However the increased penalty factor reduces the $e 2 e \quad Q o S$ (Resp. demand partitioning) which is normal, by that the increase of this factor pushes ISPs in a neutral network to minimize the difference between promised $Q o S$ and real $Q o S$, until that ISPs in this regime have for subscribers that these faithful EUs $(\mathrm{a}=500)$ for small value of $e 2 e Q o S$. Thus for non-privileged $I S P_{2}$, the increasing of this factor has more impact on this ISP compared to ISPs of neutral network, the increase of this factor may even push the EUs of this ISP to migrate to 
another ISP offering a significant $e 2 e \quad Q o S$ or to unsubscribe completely. These impacts of the increased penalty factor for the non-privileged $I S P_{2}$ have an inverse impact for the privileged $I S P_{1}$, which allows it to offer a significant $e 2 e Q o S$ and get a large market share.

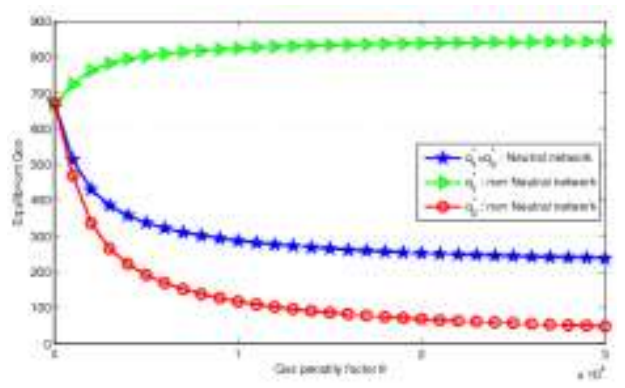

Figure 4. Impact of the Penalty Factor on Equilibrium $Q o S$

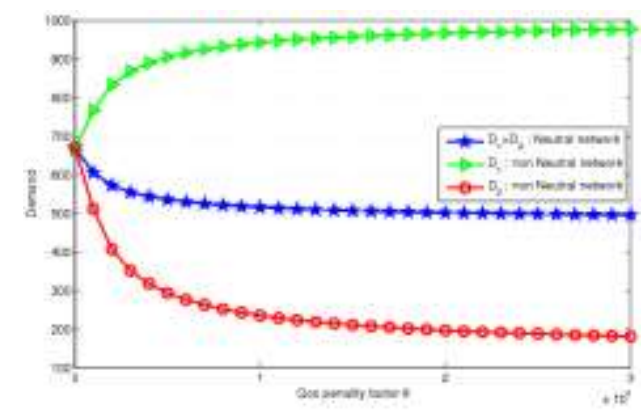

Figure 5. Impact of the Penalty Factor on Demand Partitioning

\subsection{Impact of the Available Bandwidth $\Phi_{c}$}

The Figure 6 represents the $e 2 e Q o S$ variation w.r.t the bandwidth $\Phi_{c}$, we note that for small bandwidth values between (1600 - 3000), ISPs in a neutral network or a nonprivileged $I S P_{2}$ can not choose large $e 2 e Q o S$, However, the $C P$ does not have a large bandwidth to ensure $e 2 e Q o S$ promised by the privileged $I S P_{1}$, even-if it chooses $e 2 e$ $Q o S$ as much as possible according to the $\Phi_{c}$ bandwidth arriving to choose $\tilde{q}^{\max }$, and for bandwidth values (between 3000 and 78000), the ISPs of a neutral network are increasing their $e 2 e Q o S$ in a remarkable manner with respect to the $I S P_{2}$ non-neutral network until all $I S P$ s choose $\tilde{q}^{\max }$, This is the case where the bandwidth ranges from (78000 - 200000).

The Figure 7 shows the demand partitioning between ISPs w.r.t the bandwidth $\Phi_{c}$, we note that for ISPs in a neutral network have the same demands and proportionally increase with $\Phi_{c}$ until 7800 , and demand is constant for all ISPs. For non-neutral network, and for small bandwidth values (between 1600 and 3000), ISPs in a neutral network or nonprivileged $\mathrm{ISP}_{2}$ can not choose great values of the $e 2 e Q o S$, however, the $C P$ does not have a large bandwidth to ensure promised $e 2 e Q o S$ of the privileged $I S P_{1}$, even-if this latter choose a largest $e 2 e Q o S$ possible w.r.t bandwidth, getting to choose $\tilde{q}^{\max }$, and for bandwidth values (between 3000 and 78000), the ISPs of a neutral network are 
increasing their $e 2 e Q o S$ in a remarkable manner w.r.t the $I S P_{2}$ in non-neutral network until all ISPs choose $\tilde{q}^{\max }$, it is the case of a bandwidth between 78000 and 200000.

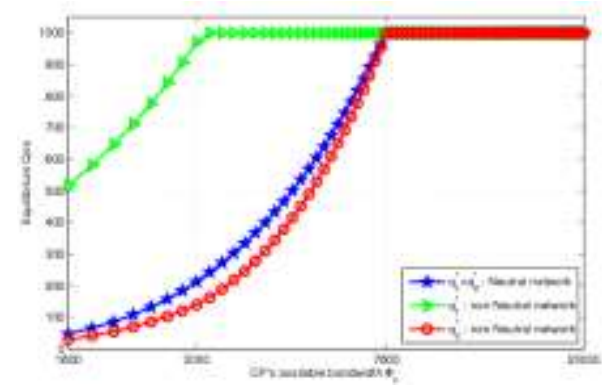

Figure 6. Impact of the Available Bandwidth $\Phi_{c}$ on Equilibrium $Q o S$

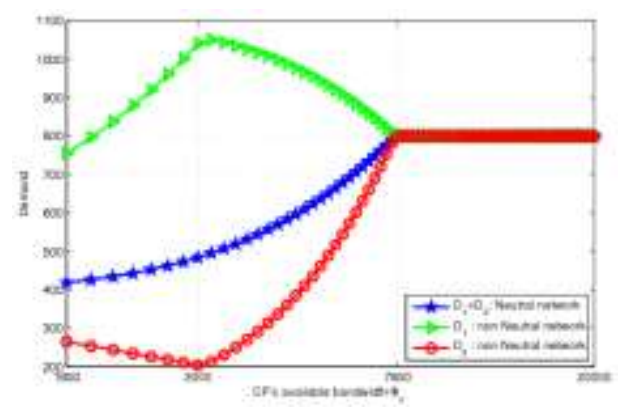

Figure 7. Impact of the Available Bandwidth $\Phi_{c}$ of the CP on Partitioning Demand

The Figure 8 represents the net revenue of the ISPs w.r.t the $\Phi_{c}$ bandwidth, in the neutral network, the ISPs equitably share the net revenue between them. However, the privileged $I S P_{1}$ (if the non-neutrality) attracts a large market share which impoverished the net revenue of the $I S P_{2}$. We remark that there are three regimes mainly due to the variation of the $e 2 e \operatorname{QoS}$ (Figure 6), well, usually when the ISPs choose $\tilde{q}^{\max }$, we note that there is a certain difference in net revenue due to the absence of penalty on quality of service, but this difference will disappear when bandwidth $\Phi_{c}$ tends to great values. Compared with the results of net revenue when price changes with fixed $e 2 e Q o S$, the results of our study will allow ISPs to achieve significant net revenue.

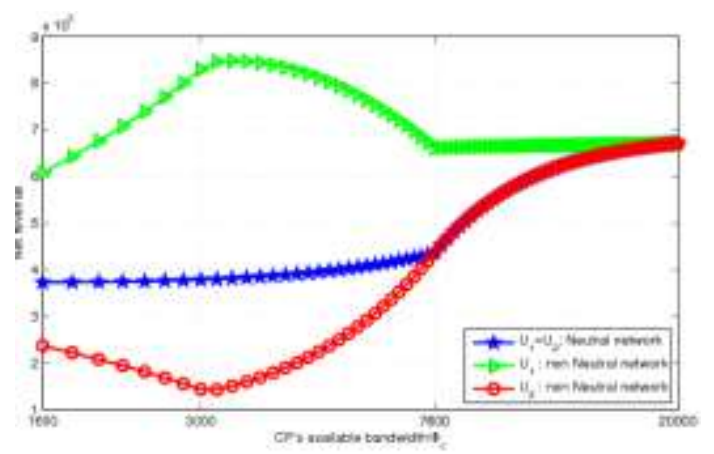

Figure 8. Impact of the Available Bandwidth $\Phi_{c}$ of the CP on the Net Revenue 


\subsubsection{Neutral Setup VS. non-neutral Setup}

To give priority to its contents over that of the $I S P_{2}$, The $I S P_{1}$ signed a special contract with the content provider. This contract can be considered as roadblocks or shortcuts defined by the $C P$ to discern the content of the $I S P_{1}$. Figures 6 and 7 illustrate respectively, the change in $e 2 e Q o S$, and the demands at the Nash equilibrium, by varying the quality of $\Phi_{c}$ bandwidth offered by $C P$. We note that the equilibrium $e 2 e$ $Q o S$ for all ISPs in a neutral network and a non-neutral network increase with $\Phi_{c}$. A special feature is that the $e 2 e Q o S$ at equilibrium of non-neutrality are more important than the $e 2 e Q o S$ in a neutral network. This encourages customers to purchase services, which explains the increase in total demand in non-neutral networks (see Figure 7). Otherwise, the $I S P_{1}$ has more power and becomes the master of the market, giving it the ability to offer services with better quality. This causes attract new subscribers, reducing demand for the $I S P_{2}$ and even its turnover. When $\Phi_{c}$ is relatively low and not sufficient to answer to the total demand, the $I S P_{2}$ without privileged contract makes less than its competitor, this comes from a lack of bandwidth was consumed by the $I S P_{1}$. We note that when the content provider manages an enormous bandwidth to answer the total demand, the measures of neutrality meet the measures of non-neutrality. Thus, the $I S P_{1}$ had no reason to invest in the signing of a privileged contract since the two regimes (neutral and non-neutral) give the same result.

\subsubsection{Non-neutrality Sustains Monopolistic and Unfair Competition}

The Figure 8 represents the net revenue of the both internet service providers for the neutral case and the non-neutral case. Due to the absence of penalty on the quality of service ( $Q O S$ since announced is encountered in non-neutral conditions), the $I S P_{1}$ attracts a higher market share. Clearly this situation-where the $I S P_{1}$ has advantages over the $I S P_{2}$ - is completely unfair. In fact, this induces a kind of monopoly position among ISPs. However, this monopoly situation implicitly prohibits the competitors from entering the market by using unfair competitive practices arising from its influence on the market as a privileged $I S P$.

\section{Impact of Competition}

\subsection{Price of Anarchy}

The price of anarchy is the ratio of the worst case objective function value of a Nash equilibrium and that of an optimal outcome. That measures how the efficiency of a system degrades due to selfish behavior of its agents. The price of anarchy [11] is a method to measure the inefficiency of equilibrium, it has been used to measure the inefficiency in congestion networks. In this case, each user of the network has a source and destination and they must pay a cost to travel from their source to their destination. In this case it is given by:

$$
\operatorname{PoA}=\frac{\min _{p, \tilde{q}} W_{N E}(p, \tilde{q})}{\max _{p, \tilde{q}} W(p, \tilde{q})}
$$


where $W(p, \tilde{q})=\sum_{i=1}^{N} U_{i}(p, \tilde{q})$ is a function of welfare and $W_{N E}(p, \tilde{q})=\sum_{i=1}^{N} U_{i}\left(p^{*}, \tilde{q}^{*}\right)$ is a sum of utilities of all actors in the Nash equilibrium.

We represent the price of anarchy variation if the price payable by ISPs is fixed (Figure 9 ). The first remark is that the price of anarchy exceeds 0.5 , it means that the equilibrium is socially acceptable for any value of bandwidth, but the price of anarchy varies according to three situations. The first when we have no enough bandwidth, the ISPs do not express their selfish behavior. The second situation where the $C P$ has a bandwidth medium, the ISPs are becoming more and more selfish, and the third one where we have a great value of the bandwidth $\Phi_{c}$, the selfish behavior weakens. But generally, neutrality is socially good for ISPs when the bandwidth $\Phi_{c}$ is low, by cons, when $\Phi_{c}$ is important, the price of anarchy of a non-neutral network is higher compared to that of the neutral network. Thus when $\Phi_{c}$ tends to great values, each case of neutrality become socially responsible.

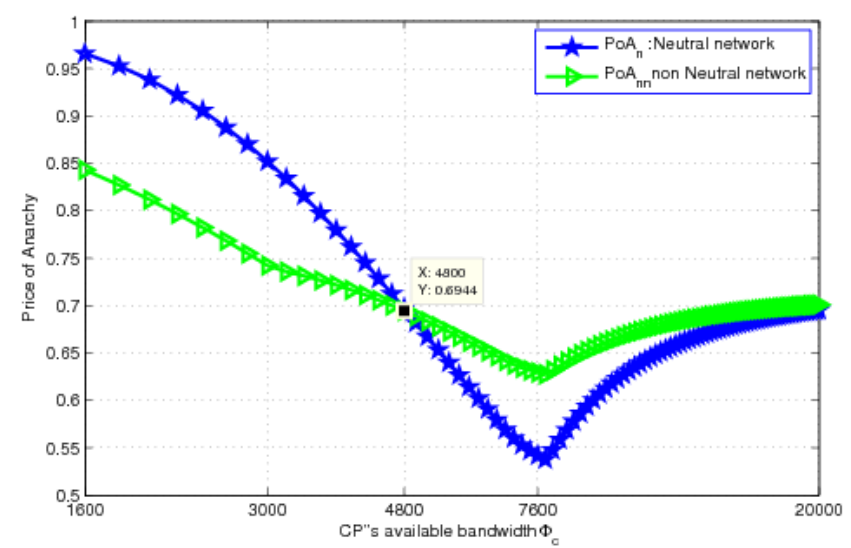

Figure 9. Impact of the Available Bandwidth $\Phi_{c}$ on the Price of Anarchy

\section{Conclusion}

We presented in this work a non-cooperative game market share. Each ISP reports some $e 2 e Q o S$ reference it claims to guarantee to its subscribers. Then, each ISP, taking into account the demand generated, determines the best $e 2 e Q o S$ maximizes its net revenue. In addition, both neutral (no discrimination on the data flowing through the network) and non-neutral when some specific ISP signed a special contract with the content provider to focus its content. Based on the Rosen's Supermodularity condition, we proved the existence and uniqueness of a Nash equilibrium for both cases. We have shown numerically that the non-neutral regime is beneficial for $E U s$ because it involves at great rates and improved quality of service. However, it can support the monopolistic and unfair competition between internet service providers.

\section{Appendix}

\subsection{Existence of the Nash Equilibrium $e 2 e Q o S$ in Neutral Network}

Equation (14) represent the second derivative of the utility function (11) w.r.t the quality of service: 


$$
\begin{aligned}
& \frac{\partial^{2}}{\partial^{2} \tilde{q}_{i}} U_{i}(p, \tilde{q})=-\frac{2 \vartheta_{i} c_{i}}{\left(1-c_{i} \tilde{q}_{i}\right)^{3}}-\frac{2 \theta_{i}\left(\beta_{i}^{i}-\sum_{k, k \neq i} \beta_{k}^{i}\right)\left(\Phi_{c}-D(p, \tilde{q})\right)^{2}\left(1-c_{i} \tilde{q}_{i}\right)\left(\Phi_{c}-D(p, \tilde{q})+\tilde{q}_{i}\right)}{\left(\tilde{q}_{i}+\left(1-c_{i} \tilde{q}_{i}\right)\left(\Phi_{c}-D\left(p, \tilde{q}_{i}\right)\right)\right)^{3}} \\
& -\frac{2 \theta_{i}\left(\left(\Phi_{c}-D(p, \tilde{q})\right)^{2}-\left(\beta_{i}^{i}-\sum_{k, k \neq i} \beta_{k}^{i}\right) \tilde{q}_{i}^{2}\right)\left(-\left(\beta_{i}^{i}-\sum_{k, k \neq i} \beta_{k}^{i}\right)\left(1-c_{i} \tilde{q}_{i}\right)-\left(\Phi_{c}-D(p, \tilde{q}) c_{i}+1\right)\right.}{\left(\tilde{q}_{i}+\left(1-c_{i} \tilde{q}_{i}\right)\left(\Phi_{c}-D(p, \tilde{q})\right)\right)^{3}}
\end{aligned}
$$

and we have,

$$
1-\left(\Phi_{c}-D(p, \tilde{q})\right) c_{i}>0
$$

So,

$$
\frac{\partial^{2}}{\partial^{2} \tilde{q}_{i}} U_{i}(p, \tilde{q})<-\frac{2 \theta_{i}\left(\beta_{i}^{i}-\sum_{k, k \neq i} \beta_{k}^{i}\right)\left(\Phi_{c}-D(p, \tilde{q})\right)\left(1-c_{i} \tilde{q}_{i}\right)\left(\Phi_{c}-D(p, \tilde{q})+\tilde{q}_{i}\right)\left(\Phi_{c}-D(p, \tilde{q})-1\right)}{\left(\tilde{q}_{i}+\left(1-c_{i} \tilde{q}_{i}\right)\left(\Phi_{c}-D\left(p, \tilde{q}_{i}\right)\right)\right)^{3}}
$$

Then

$$
\frac{\partial^{2}}{\partial^{2} \tilde{q}_{i}} U_{i}(p, \tilde{q})<0
$$

The second derivative of the utility function is negative, then the utility function is concave, hence the existence of the Nash equilibrium $e 2 e$ QoS follows, [20].

\subsection{Uniqueness of the Nash Equilibrium $e 2 e Q o S$ in Neutral Network}

The uniqueness of the equilibrium point is guaranteed if the utility function satisfies the conditions of Rosen [20]. Moulin [8], derived from the supermodularity condition, which is another alternative to satisfy the conditions of Rosen: The Nash equilibrium point is unique if:

$$
-\frac{\partial^{2}}{\partial^{2} \tilde{q}_{i}} U_{i}(p, \tilde{q})-\sum_{j, j \neq i}\left|\frac{\partial^{2}}{\partial \tilde{q}_{i} \partial \tilde{q}_{j}} U_{i}(p, \tilde{q})\right|>0
$$

the mixed partial derivative is given by:

$$
\frac{\partial^{2}}{\partial \tilde{q}_{i} \partial \tilde{q}_{j}} U_{i}(p, \tilde{q})=-\frac{2 \theta_{i} \tilde{q}_{i}\left(\beta_{j}^{j}-\sum_{k, k \neq j} \beta_{k}^{j}\right)\left(\Phi_{c}-D(p, \tilde{q})+\tilde{q}_{i}\left(1-c_{i} \tilde{q}_{i}\right)\left(\beta_{i}^{i}-\sum_{k, k \neq i} \beta_{k}^{i}\right)\right)}{\left(\tilde{q}_{i}+\left(1-c_{i} \tilde{q}_{i}\right)\left(\Phi_{c}-D(p, \tilde{q})\right)\right)^{3}}<0
$$

we pose

we verify that:

$$
A=-\frac{\partial^{2}}{\partial^{2} \tilde{q}_{i}} U_{i}(p, \tilde{q})-\sum_{j, j \neq i}\left|\frac{\partial^{2}}{\partial \tilde{q}_{i} \partial \tilde{q}_{j}} U_{i}(p, \tilde{q})\right|
$$

$$
A>0
$$

From the progression A, and after substitution of (14) and (17) in (16), we have:

$A=\frac{2 \vartheta_{i} c_{i}}{\left(1-c_{i} \tilde{q}_{i}\right)^{3}}+\frac{2 \theta_{i}\left(\beta_{i}^{i}-\sum_{k, k \neq i} \beta_{k}^{i}\right)\left(\Phi_{c}-D(p, \tilde{q})\right)^{2}\left(1-c_{i} \tilde{q}_{i}\right)\left(\Phi_{c}-D(p, \tilde{q})+\tilde{q}_{i}\right)}{\left(\tilde{q}_{i}+\left(1-c_{i} \tilde{q}_{i}\right)\left(\Phi_{c}-D\left(p, \tilde{q}_{i}\right)\right)\right)^{3}}-$ 


$$
\begin{aligned}
& \frac{2 \theta_{i}\left(\left(\Phi_{c}-D(p, \tilde{q})\right)^{2}-\left(\beta_{i}^{i}-\sum_{k, k \neq i} \beta_{k}^{i}\right) \tilde{q}_{i}^{2}\right)\left(\left(\beta_{i}^{i}-\sum_{k, k \neq i} \beta_{k}^{i}\right)\left(1-c_{i} \tilde{q}_{i}\right)+\left(\Phi_{c}-D(p, \tilde{q})\right) c_{i}-1\right)}{\left(\tilde{q}_{i}+\left(1-c_{i} \tilde{q}_{i}\right)\left(\Phi_{c}-D(p, \tilde{q})\right)\right)^{3}} \\
& -\left(\frac{2 \theta_{i} \tilde{q}_{i}\left(\Phi_{c}-D(p, \tilde{q})+\tilde{q}_{i}\left(1-c_{i} \tilde{q}_{i}\right)\left(\beta_{i}^{i}-\sum_{k, k \neq i} \beta_{k}^{i}\right)\right)}{\left(\tilde{q}_{i}+\left(1-c_{i} \tilde{q}_{i}\right)\left(\Phi_{c}-D(p, \tilde{q})\right)\right)^{3}}\right) \sum_{j, j \neq i}\left(\beta_{j}^{j}-\sum_{k, k \neq j} \beta_{k}^{j}\right)
\end{aligned}
$$

on the assumption 3, we have: $\beta_{i}^{i}-\sum_{j, j \neq i} \beta_{i}^{j} \leq \frac{1}{N}, \forall i, j=1, \ldots, N$., then we have:

$$
\begin{gathered}
A>\frac{2 \vartheta_{i} c_{i}}{\left(1-c_{i} \tilde{q}_{i}\right)^{3}}+\frac{2 \theta_{i}\left(\Phi_{c}-D(p, \tilde{q})+\tilde{q}_{i}\right)\left(\left(\Phi_{c}-D(p, \tilde{q})\right)\left(\Phi_{c}-D(p, \tilde{q})\right)\left(\frac{\left(1-c_{i} \tilde{q}_{i}\right)}{N}\right)-\tilde{q}_{i}\right)}{\left(\tilde{q}_{i}+\left(1-c_{i} \tilde{q}_{i}\right)\left(\Phi_{c}-D\left(p, \tilde{q}_{i}\right)\right)\right)^{3}} \\
-\frac{2 \theta_{i}\left(\Phi_{c}-D(p, \tilde{q})+\tilde{q}_{i}\right)\left(\Phi_{c}-D(p, \tilde{q})-\tilde{q}_{i}\right)\left(\frac{\left(1-c_{i} \tilde{q}_{i}\right)}{N}\right)}{\left(\tilde{q}_{i}+\left(1-c_{i} \tilde{q}_{i}\right)\left(\Phi_{c}-D(p, \tilde{q})\right)\right)^{3}}
\end{gathered}
$$

with

$$
\Phi_{c}-D(p, \tilde{q})=\frac{\tilde{q}_{i}}{\left(1-c_{i} \tilde{q}_{i}\right)}+\sum_{k, k \neq i} \frac{\tilde{q}_{k}}{\left(1-c_{k} \tilde{q}_{k}\right)}
$$

then,

$$
\begin{array}{r}
A>\frac{2 \vartheta_{i} c_{i}}{\left(1-c_{i} \tilde{q}_{i}\right)^{3}}+\frac{2 \theta_{i} \tilde{q}_{i}\left(\Phi_{c}-D(p, \tilde{q})+\tilde{q}_{i}\right)\left(\frac{\Phi_{c}-D(p, \tilde{q})}{N}-1\right)}{\left(\tilde{q}_{i}+\left(1-c_{i} \tilde{q}_{i}\right)\left(\Phi_{c}-D\left(p, \tilde{q}_{i}\right)\right)\right)^{3}} \\
+\frac{2 \theta_{i}\left(\Phi_{c}-D(p, \tilde{q})+\tilde{q}_{i}\right)\left(1-c_{i} \tilde{q}_{i}\right)\left(\frac{\Phi_{c}-D(p, \tilde{q})}{N}\right)\left(\sum_{k, k \neq i} \frac{\tilde{q}_{k}}{\left(1-c_{k} \tilde{q}_{k}\right)}-1\right)}{\left(\tilde{q}_{i}+\left(1-c_{i} \tilde{q}_{i}\right)\left(\Phi_{c}-D(p, \tilde{q})\right)\right)^{3}}
\end{array}
$$

For $\quad \tilde{q}_{i} \frac{1}{1+c_{i}} ; 1 \quad$ we $\quad$ have: $\quad \frac{\tilde{q}_{i}}{\left(1-c_{i} \tilde{q}_{i}\right)} 1$ which implies that $\Phi_{c}-D(p, \tilde{q})=\sum_{k} \frac{\tilde{q}_{i}}{\left(1-c_{i} \tilde{q}_{i}\right)} \quad N$

Finally we have:

$$
A=-\frac{\partial^{2}}{\partial^{2} \tilde{q}_{i}} U_{i}(p, \tilde{q})-\sum_{j, j \neq i}\left|\frac{\partial^{2}}{\partial \tilde{q}_{i} \partial \tilde{q}_{j}} U_{i}(p, \tilde{q})\right|>0
$$

Regarding the assumption 1 and according to the remark 1 , gives the positivity of the last expression. This means that the supermodularity condition of Moulin holds, then this game satisfies the conditions of Rosen, where the uniqueness of the equilibrium is verified following, [8]. 


\subsection{Existence and Uniqueness of the Nash equilibrium $e 2 e \quad Q o S$ in Non Neutral Network}

It is clear that the expression of the utility function of ISPs who have no privileged contract with the $C P$, is the same as that of ISPs in the case of net neutrality, namely, equation (11). Now the utility function of these ISPs, satisfies the properties of the concavity and uniqueness, equation (16). Therefore, to prove the existence and uniqueness of equilibrium under conditions of non-neutrality, it suffices to prove that the utility function of the $I S P_{1}(12)$, verifies the concavity properties (existence) and the state of supermodularity, equation (16), (Uniqueness).

\subsubsection{Existence}

Equation (21) represents the second derivative of the utility function of $I S P_{1}$ :

$\frac{\partial^{2}}{\partial^{2} \tilde{q}_{1}} U_{1}(p, \tilde{q})=-\frac{\left.2 \vartheta_{1}\left(\Phi_{c}-D_{1}(p, \tilde{q})\right)-\beta_{1}^{1^{2}} \tilde{q}_{1}\right)}{\left.\left(\Phi_{c}-D_{1}(p, \tilde{q})\right)-\tilde{q}_{1}\right)^{2}}-\frac{2 \vartheta_{1} \tilde{q}_{1}\left(\beta_{1}^{1}\left(\Phi_{c}-D_{1}(p, \tilde{q})+1\right)\right)^{2}}{\left.\left(\Phi_{c}-D_{1}(p, \tilde{q})\right)-\tilde{q}_{1}\right)^{3}}$

Since we have $\tilde{q}_{1}=\frac{1}{\operatorname{Delay}_{u}^{1}+c_{1}}$, then $1-\tilde{q}_{1} c_{1} \geq 0$, and, $c_{1}=\frac{1}{\Phi_{c}-D_{1}(p, \tilde{q})}$, we notice that, $\Phi_{c}-D_{1}(p, \tilde{q})-\tilde{q}_{1} \geq 0$. Then it is easy to note (Equation (21)) that:

$$
\frac{\partial^{2}}{\partial^{2} \widetilde{q}_{1}} U_{1}(p, \tilde{q})<0
$$

Thus, the utility function is concave, thereafter, the existence of a Nash equilibrium is the result of [20].

\subsubsection{Uniqueness}

Similarly, to show the uniqueness of the equilibrium point, we need to verify if $U_{1}^{I S P}(p, \tilde{q})$ in the equation (21) satisfied the supermodularity condition, namely, equation(16). However, we have:

$$
\frac{\partial^{2}}{\partial \tilde{q}_{1} \partial \tilde{q}_{l}} U_{1}(p, \tilde{q})=\frac{2 \vartheta_{1} \tilde{q}_{1} \beta_{1}^{l}\left(\Phi_{c}-D_{1}(p, \tilde{q})+\tilde{q}_{1} \beta_{1}^{1}\right)}{\left(\Phi_{c}-D_{1}(p, \tilde{q})-\tilde{q}_{1}\right)^{3}}>0
$$

Assume that:

$$
B=-\frac{\partial^{2}}{\partial^{2} \tilde{q}_{1}} U_{1}(p, \tilde{q})-\sum_{l, l \neq 1}\left|\frac{\partial^{2}}{\partial \tilde{q}_{1} \partial \tilde{q}_{l}} U_{1}(p, \tilde{q})\right|
$$

We verify that:

$$
B>0
$$

then

$$
\begin{aligned}
& B=\frac{\left.2 \vartheta_{1}\left(\Phi_{c}-D_{1}(p, \tilde{q})\right)-\beta_{1}^{12} \tilde{q}_{1}\right)}{\left.\left(\Phi_{c}-D_{1}(p, \tilde{q})\right)-\tilde{q}_{1}\right)^{2}}+\frac{2 \vartheta_{1} \tilde{q}_{1}\left(\beta_{1}^{1}\left(\Phi_{c}-D_{1}(p, \tilde{q})+1\right)\right)^{2}}{\left.\left(\Phi_{c}-D_{1}(p, \tilde{q})\right)-\tilde{q}_{1}\right)^{3}}-\sum_{l, l \neq 1}\left(\frac{2 \vartheta_{1} \tilde{q}_{1} \beta_{1}^{l}\left(\Phi_{c}-D_{1}(p, \tilde{q})+\tilde{q}_{1} \beta_{1}^{1}\right)}{\left(\Phi_{c}-D_{1}(p, \tilde{q})-\tilde{q}_{1}\right)^{3}}\right) \\
& \left.\left.>\frac{2 \vartheta_{1}}{\left.\left(\Phi_{c}-D_{1}(p, \tilde{q})\right)-\tilde{q}_{1}\right)^{3}}\left[\left(\Phi_{c}-D_{1}(p, \tilde{q})\right)\right)\left(\Phi_{c}-D_{1}(p, \tilde{q})\right)+\left(1-\beta_{1}^{1^{2}}\right) \tilde{q}_{1}\right)+\tilde{q}_{1}^{2} \beta_{1}^{1}\left(\beta_{1}^{1}-\sum_{l, l \neq 1} \beta_{1}^{l}\right)\right]
\end{aligned}
$$


$+\frac{2 \vartheta_{1} \tilde{q}_{1} \beta_{1}^{1}\left(\Phi_{c}-D_{1}(p, \tilde{q})\right)}{\left.\left(\Phi_{c}-D_{1}(p, \tilde{q})\right)-\tilde{q}_{1}\right)^{3}}\left[\beta_{1}^{1}-\sum_{l, l \neq 1} \beta_{1}^{l}\right]>0$

So

$$
-\frac{\partial^{2}}{\partial^{2} \tilde{q}_{1}} U_{1}(p, \tilde{q})-\sum_{l, l \neq 1}\left|\frac{\partial^{2}}{\partial \tilde{q}_{1} \partial \tilde{q}_{l}} U_{1}(p, \tilde{q})\right|>0 .
$$

This result shows that for the $I S P_{1}$ utility function, the supermodularity condition of Moulin is satisfied, hence the uniqueness of equilibrium.

\section{References}

[1] O. Andrew, "Network neutrality, search neutrality, and the never-ending conflict between efficiency and fairness in markets", Review of Network Economics, vol. 8, no. 1, (2009), pp. 1-21.

[2] M. Baslam, L. Echabbi, R. El-Azouzi and E. Sabir, "Joint price and qos market share game with adversarial service providers and migrating customers", International Conference on Game Theory for Networks, (2011), pp. 642-657.

[3] M. Baslam, R. El-Azouzi, E. Sabir and L. Echabbi, "Market share game with adversarial access providers: A neutral and a non-neutral network analysis", Network Games, Control and Optimization (NetGCooP), 2011 5th International Conference, (2011), pp. 1-6.

[4] M. Baslam, R. El-Azouzi, E. Sabir, L. Echabbi and E. H. Bouyakhf, "A game theoretic analysis of priceQoS market share in presence of adversarial service providers", Game theory relaunched, InTech, (2013).

[5] N. Economides and J. TÃ $¥ g$, "Network neutrality on the Internet: A two-sided market analysis", Information Economics and Policy, vol. 24, no. 2, (2012), pp. 91-104.

[6] R. El Azouzi, E. Altman and L. Wynter, "Telecommunications network equilibrium with price and quality-of-service characteristics", Teletraffic Science and Engineering, vol. 5, (2003), pp. 369-378.

[7] D. Fudenberg and J. Tirole, "Game Theory", The MIT Press, Cambridge, Massachusetts, (1991).

[8] D. Gabay and H. Moulin, "On the uniqueness and stability of Nash's equilibrium in non cooperative games”, Université Paris IX-Dauphine, Centre de recherche de mathématiques de la décision, (1978).

[9] H. Garmani, M. Baslam and M. Jourhmane, "Caching Games between ISP in Information Centric Network", International Journal of Control and Automation, vol. 11, no. 4, (2018), pp. 125-142.

[10] L. Kleinrock, "Queueing systems, volume 2: Computer applications", Wiley New York, vol. 66, (1976).

[11] E. Koutsoupias and C. Papadimitriou, "Worst-case equilibria", Annual Symposium on Theoretical Aspects of Computer Science, (1999), pp. 404-413.

[12] T. M. Lenard and R. J. May, "Net Neutrality or Net Neutering: Should Broadband Internet Services be Regulated", Springer Science \& Business Media, (2006).

[13] P. Maillé, P. Reichl and B. Tuffin, "Internet governance and economics of network neutrality", Telecommunication Economics, (2012), pp. 108-116.

[14] P. Maillé, G. Simon and B. Tuffin, "Toward a net neutrality debate that conforms to the 2010s", IEEE communications magazine, vol. 54, no. 3, (2016), pp. 94-99.

[15] P. Maillé and B. Tuffin, "Non-neutrality Pushed by Big Content Providers", International Conference on the Economics of Grids, Clouds, Systems, and Services, (2017), pp. 29-39.

[16] P. Maillé and B. Tuffin, "Telecommunication network economics: from theory to applications", Cambridge University Press, (2014).

[17] E. Maskin, "Nash equilibrium and welfare optimality", The Review of Economic Studies, vol. 66, no. 1, (1999), pp. 23-38.

[18] J. Musacchio, G. Schwartz and J. Walrand, "A two-sided market analysis of provider investment incentives with an application to the net-neutrality issue", Review of Network Economics, vol. 8, no. 1, (2009).

[19] P. Njoroge, A. Ozdaglar, N. E. Stier-Moses and G. Y. Weintraub, "Investment in two-sided markets and the net neutrality debate", Review of Network Economics, vol. 12, no. 4, (2013), pp. 355-402.

[20] J. B. Rosen, "Existence and uniqueness of equilibrium points for concave n-person games" Econometrica: Journal of the Econometric Society, (1965), pp. 520-534.

[21] J. Tirole, "The theory of industrial organization", MIT press, (1988).

[22] C. Zhang, B. Gu, Z. Liu, K. Yamori and Y. Tanaka, "A stackelberg game based analysis for interactions among Internet service provider, content provider, and advertisers", Consumer Communications \& Networking Conference (CCNC), 2017 14th IEEE Annual, (2017), pp. 231-235. 